\title{
Some New Algorithms for High-Precision Computation of Euler's Constant
}

\author{
By Richard P. Brent* and Edwin M. McMillan
}

\begin{abstract}
We describe several new algorithms for the high-precision computation of Euler's constant $\gamma=0.577 \ldots$ Using one of the algorithms, which is based on an identity involving Bessel functions, $\gamma$ has been computed to 30,100 decimal places. By computing their regular continued fractions we show that, if $\gamma$ or $\exp (\gamma)$ is of the form $P / Q$ for integers $P$ and $Q$, then $|Q|>10^{15000}$.
\end{abstract}

1. Introduction. Euler's constant $\gamma$ is defined by

$$
\gamma=\lim _{m \rightarrow \infty}\left(H_{m}-\ln (m)\right)
$$

where $H_{m}=\sum_{k=1}^{m} 1 / k$.

Recently $\zeta(3)$ was proved irrational [17] with the aid of a rapidly converging continued fraction, and conceivably a similar method might be used to prove the irrationality of $\gamma$. Thus, there is some interest in finding rapidly converging expressions for $\gamma$. We give several such expressions below.

Early computations of $\gamma$ used the Euler-Maclaurin expansion to accelerate convergence of (1): see Brent [7] and Glaisher [12]. Sweeney [16] suggested a method which avoids the need for computation of the Bernoulli numbers which appear in the Euler-Maclaurin expansion, and Brent [7], [8] used Sweeney's method to compute $\gamma$ to 20,700 decimal places. In Section 3 we describe an algorithm which is about twice as fast as Sweeney's. The algorithm depends on some identities, given in Section 2 , involving modified Bessel functions. To demonstrate the effectiveness of the algorithm we have used it to compute $\gamma$ to 30,100 decimal places; see Section 5. Some other algorithms for the high-precision computation of $\gamma$ are briefly described and compared in Section 4.

2. Some Bessel Function Identities. The modified Bessel functions $I_{\nu}(z)$ and $K_{0}(z)$ are defined by

$$
I_{\nu}(z)=\sum_{k=0}^{\infty} \frac{(z / 2)^{\nu+2 k}}{k ! \Gamma(\nu+k+1)} \quad \text { and } \quad K_{0}(z)=-\partial I_{\nu}(z) /\left.\partial \nu\right|_{\nu=0} .
$$

Received January 22, 1979; revised May 15, 1979.

AMS (MOS) subject classifications (1970). Primary 10-04, 10A40; Secondary 10F20, 10F35, 65A05, 68A20.

Key words and phrases. Euler's constant, Mascheroni's constant, gamma, Bessel functions, rational approximation, regular continued fractions, multiple-precision arithmetic, Gauss-Kusmin law.

* The work of the first author was supported in part by National Science Foundation grant 1-442427-21164-2 at the University of California, Berkeley. This work was also supported by the U. S. Department of Energy under Contract W-7405-ENG-48. 
It is easy to verify, by carrying out the differentiation indicated above, that

$$
\gamma+\ln (z / 2)=\frac{S_{0}(z)-K_{0}(z)}{I_{0}(z)}
$$

where

$$
S_{0}(z)=\sum_{k=0}^{\infty} \frac{(z / 2)^{2 k}}{(k !)^{2}} H_{k} .
$$

For real positive $z, K_{0}(z)$ and $I_{0}(z)$ have the asymptotic expansions

$$
K_{0}(z) \sim\left(\frac{\pi}{2 z}\right)^{1 / 2} e^{-z} \sum_{k=0}^{\infty}(-1)^{k} a_{k}(z)
$$

and

$$
I_{0}(z) \sim(2 \pi z)^{-1 / 2} e^{z} \sum_{k=0}^{\infty} a_{k}(z)
$$

where

$$
a_{k}(z)=\frac{1^{2} 3^{2} \cdots(2 k-1)^{2}}{k !(8 z)^{k}}=\frac{[(2 k) !]^{2}}{(k !)^{3}(32 z)^{k}} .
$$

A proof is given in Watson [19, Section 7.23].

For real $z \geqslant 1$, the first terms in the asymptotic expansions (3) and (4) give upper and lower bounds on $K_{0}(z)$ and $I_{0}(z)$, respectively:

$$
0<K_{0}(z)<\left(\frac{\pi}{2 z}\right)^{1 / 2} e^{-z} \text { and } I_{0}(z)>(2 \pi z)^{-1 / 2} e^{z} \text {. }
$$

Thus, taking $z=2 n \geqslant 2$ in (2), we have

$$
0<U(n) / V(n)-\gamma=K_{0}(2 n) / I_{0}(2 n)<\pi e^{-4 n},
$$

where

$$
U(n)=S_{0}(2 n)-I_{0}(2 n) \ln (n)=\sum_{k=0}^{\infty}\left(\frac{n^{k}}{k !}\right)^{2}\left(H_{k}-\ln (n)\right)
$$

and

$$
V(n)=I_{0}(2 n)=\sum_{k=0}^{\infty}\left(\frac{n^{k}}{k !}\right)^{2}
$$

In the following section we describe an algorithm (B1) for computing $\gamma$ using (5) to (7). It is interesting to note that the relations (2) to (4) were essentially given by Riemann [14] in 1855 , but the possibility of using them to compute Euler's constant appears to have been overlooked.

3. The Algorithm B1. Suppose we wish to evaluate $\gamma$ to $d$ decimal places. If we choose

$$
n=\lfloor c+1 / 4 \ln (10) d\rfloor
$$


for some suitable constant $c$ then, from (5),

$$
|\gamma-U(n) / V(n)|<\pi e^{4-4 c} 10^{-d} .
$$

Thus, we need only evaluate $U(n)$ and $V(n)$ sufficiently accurately, and then perform one high-precision division, to evaluate $\gamma$ to the required accuracy.

Let

$$
\begin{array}{ll}
A_{k}=\left(\frac{n^{k}}{k !}\right)^{2}\left(H_{k}-\ln (n)\right), & U_{k}=\sum_{j=0}^{k} A_{j} \\
B_{k}=\left(\frac{n^{k}}{k !}\right)^{2}, & V_{k}=\sum_{j=0}^{k} B_{j} .
\end{array}
$$

Then

$$
A_{0}=-\ln (n), \quad B_{0}=1, \quad U_{0}=A_{0}, \quad V_{0}=1,
$$

and for $k=1,2, \ldots$, we have

$$
\begin{array}{ll}
B_{k}=B_{k-1} n^{2} / k^{2}, & A_{k}=\left(A_{k-1} n^{2} / k+B_{k}\right) / k, \\
U_{k}=U_{k-1}+A_{k}, & V_{k}=V_{k-1}+B_{k} .
\end{array}
$$

For Algorithm B1, $n$ is chosen according to (8), and working precision equivalent to slightly more than $d$ (floating) decimal places is used. $\ln (n)$ is computed, e.g. by the $O\left(d^{2}\right)$ method of [9], and $A_{0}, B_{0}, U_{0}$ and $V_{0}$ are initialized as in (9). The iteration (10) is terminated when, to the working precision, $U_{k}=U_{k-1}$ and $V_{k}=$ $V_{k-1}$. The storage required is $O(d)$ as $B_{k}$ can overwrite $B_{k-1}$, etc.

For $j \geqslant 0$, let $\alpha_{j}$ be the real positive root of

$$
\alpha_{j} \ln \alpha_{j}-\alpha_{j}=j
$$

Thus, $\alpha_{0}=e \simeq 2.78, \alpha_{1} \simeq 3.59, \alpha_{2} \simeq 4.32, \alpha_{3} \simeq 4.97$, etc.

Using Stirling's approximation, we see that the number of iterations of (10) required is

$$
K=\alpha_{1} n+O(\ln (n))=1 / 4 \alpha_{1} \ln (10) d+O(\ln (d)) \simeq 2.07 d .
$$

In analyzing the time required by Algorithm B1 and other algorithms described in Section 4, we make the following simplifying assumptions.

(a) Only the time required for the inner loop(s) is considered. (The computation of $\ln (n)$ is common to all the algorithms considered, so the time required for this is neglected. The final division of $U_{K}$ by $V_{K}$ takes time $O\left(d^{2}\right)$ if done as in [9], but the constant factor is relatively small, and $o\left(d^{2}\right)$ methods exist [5].)

(b) Multiplication or division of a multiple-precision number (e.g. $A_{k-1}$ ) by a small integer (e.g. $n^{2}$ or $k$ ) takes time $d$ units. In the analysis (though not in the implementation of the algorithm) the possibility of reducing the working precision (e.g. for $A_{k}$ and $B_{k}$ when $k \simeq K$ ) is neglected. Considering this possibility complicates the analysis but is unlikely to alter the ranking of the algorithms discussed below. 
(c) Addition of two multiple-precision numbers takes time $d$ units. (The constant is assumed to be the same as for (b). Again, this is unlikely to change the ranking of the algorithms.)

Using these assumptions, each iteration of $(10)$ requires time $8 d$ (for 3 multipleprecision additions, 2 multiplications and 3 divisions by small integers). Thus, from (12), the time required by method $\mathrm{B} 1$ is about $2 \alpha_{1} \ln (10) d^{2} \simeq 16.5 d^{2}$.

It is important to note that we avoid keeping $H_{k}$ or $\left(H_{k}-\ln (n)\right)$ as a multipleprecision number and multiplying by $B_{k}$ in the inner loop. This would lead to a method with time $\Omega\left(d^{3}\right)$ if the classical multiplication algorithm were used as in [9] . The idea of using the Bessel function identities to compute $\gamma$ was suggested by the second author, and the $O\left(d^{2}\right)$ implementation was discovered by the first author.

If terms in the sum (15) are grouped as in [5] and the Schönhage-Strassen fast multiplication algorithm [15] is used, it is possible to compute $\gamma$ with error bounded by $10^{-d}$ in time $O\left\{d[\ln (d)]^{3} \ln [\ln (d)]\right\}$, asymptotically faster than any of the $\Omega\left(d^{2}\right)$ algorithms considered here. However, such "fast" algorithms are very difficult to implement and are slower than Algorithm B1 unless $d$ is very large. Thus, we do not consider them further.

4. Related Algorithms. In this section we briefly describe and compare several closely related algorithms for the computation of $\gamma$.

4.1. Algorithm B2. From (2) we have

$$
\gamma+\ln (n)=\frac{S_{0}(2 n)-K_{0}(2 n)}{I_{0}(2 n)}
$$

and from (3)

$$
K_{0}(2 n)=1 / 2(\pi / n)^{1 / 2} e^{-2 n} \sum_{k=0}^{4 n}(-1)^{k} a_{k}(2 n)+O\left(e^{-6 n} / n\right) .
$$

Thus, we can find $\gamma$ with error $O\left(n^{-1 / 2} e^{-8 n}\right)$ if $K_{0}(2 n)$ is approximated using (13). If $e^{2 n}$ is computed using the Taylor series, and the time required to compute $(\pi / n)^{1 / 2}$ is neglected, the time required by this method (B2) is about

$$
\left[\left(8 \alpha_{3}+3 \alpha_{1}+6\right) \ln (10) / 8\right] d^{2} \simeq 16.3 d^{2},
$$

not appreciably less than for the simpler method B1.

4.2. Algorithm B3. To avoid the computation of $(\pi / n)^{1 / 2} e^{-2 n}$ in (13), we may use the asymptotic series [1, Eq. (9.7.5)]

$$
I_{0}(2 n) K_{0}(2 n) \sim \frac{1}{4 n} \sum_{k=0}^{k^{\prime}} \frac{[(2 k) !]^{3}}{(k !)^{4}(16 n)^{2 k}}
$$

with $k^{\prime} \leqslant 2 n$. Empirical evidence suggests that the relative error in (14) with $k^{\prime}=2 n$ is $O\left(n^{-1 / 2} e^{-4 n}\right)$, but we have not been able to prove this. Assuming this error bound, the time required with $k^{\prime}=2 n$ is about

$$
\left(\alpha_{3}+3 / 8\right) \ln (10) d^{2} \simeq 12.3 d^{2} .
$$


This is less than the estimate $16.5 d^{2}$ for Algorithm B1, but we preferred to use B1 because of its simplicity and the difficulty in rigorously bounding the error in (14).

4.3. Exponential Integral Methods. Several algorithms are based on the identity

$$
\gamma+\ln (n)=Q(n)-R(n)
$$

where

$$
Q(n)=\int_{0}^{n}\left(\frac{1-e^{-x}}{x}\right) d x=\sum_{k=1}^{\infty} \frac{n^{k}(-1)^{k-1}}{k ! k}
$$

and

$$
R(n)=\int_{n}^{\infty} \frac{e^{-x}}{x} d x=O\left(e^{-n} / n\right)
$$

Beyer and Waterman [3], [4] took $n \simeq \ln (10) d$, worked to precision equivalent to $2 d$ decimal places to compensate for cancellation in the sum for $Q(n)$, and neglected $R(n)$. The time required for this method is about $6 \alpha_{1} \ln (10) d^{2} \simeq 49.6 d^{2}$, or three times that for method B1.

Following the suggestion of Sweeney [16], Brent [7], [8] took $n \simeq 1 / 2 \ln (10) d$, summed the series for $Q(n)$ using the equivalent of $3 d / 2$ decimal places, and approximated $R(n)$ by its asymptotic expansion

$$
R(n)=\frac{e^{-n}}{n} \sum_{k=0}^{n-2} \frac{k !}{(-n)^{k}}+O\left(e^{-2 n} / n\right) .
$$

Assuming the power series is used to compute $e^{n}$, the time required for this method is about

$$
\frac{3}{4}\left(3 \alpha_{2}+\alpha_{0}+1\right) \ln (10) d^{2} \simeq 28.8 d^{2}
$$

about twice as much as for method B1. Actual running times confirm this ratio.

Using the identity

$$
e^{n} Q(n)=\sum_{k=0}^{\infty} H_{k} \frac{n^{k}}{k !},
$$

we can evaluate $Q(n)$ by computing $\Sigma H_{k} n^{k} / k$ ! and $\Sigma n^{k} / k$ ! by recurrences similar to (10). Because all the terms in the two sums are positive, there is no need to increase the working precision to much more than the equivalent of $d$ decimal places. If $n$ $\simeq \ln (10) d$, and $R(n)$ is neglected, the time required is about $7 \alpha_{0} \ln (10) d^{2} \simeq 43.8 d^{2}$, slightly less than for Beyer and Waterman's method. If the asymptotic series (16) is used for $R(n)$, the time required is about

$$
1 / 4\left(14 \alpha_{1}+3\right) \ln (10) d^{2} \simeq 30.7 d^{2}
$$

slightly greater than for Sweeney's method.

Instead of using the asymptotic expansion (16) for $R(n)$, we could use Euler's continued fraction $[18$, p. 350$]$

$$
e^{n} R(n)=1 / n+1 / 1+1 / n+2 / 1+2 / n+3 / 1+3 / n+\cdots
$$


and the forward or backward recurrence relations. This has the advantage that $R(n)$ can be evaluated as accurately as desired, whereas with the asymptotic expansion (16) the error is $\Omega\left(e^{-2 n} / n\right)$. The choice of the optimal $n$ and the optimal number of terms in the continued fraction (evaluated by the backward recurrence relations) gives a method competitive with Algorithm B1, but much more complicated.

4.4. A Generalization. For fixed $p>0$, it follows from (1) that

$$
\gamma=\lim _{n \rightarrow \infty} \frac{\sum_{k=0}^{\infty}\left(n^{k} / k !\right)^{p}\left(H_{k}-\ln (n)\right)}{\sum_{k=0}^{\infty}\left(n^{k} / k !\right)^{p}} .
$$

With $p=1$ we obtain essentially one of the exponential integral methods mentioned above, with error $O\left(e^{-n} / n\right)$. With $p=2$ we obtain method $\mathrm{B} 1$, with error $O\left(e^{-4 n}\right)$.

We shall sketch how the error in (17) may be estimated for integer $p \geqslant 2$. Let $y(z)$ be a function of the real variable $z$, and $L$ the operator defined by $L y=z(d y / d z)$. Then

$$
V_{p}(z)=\sum_{k=0}^{\infty}(z / k !)^{p}
$$

and

$$
U_{p}(z)=\sum_{k=0}^{\infty}\left(H_{k}-\ln (z)-\gamma\right)\left(z^{k} / k !\right)^{p}
$$

are independent solutions of

$$
\left(L^{p}-(p z)^{p}\right) y=0
$$

To verify this for $y=U_{p}$, let

$$
V_{p, \nu}(z)=\sum_{k=0}^{\infty} \frac{z^{k p+\nu}}{(k !)^{p-1} \Gamma(k+\nu+1)} .
$$

Then it is easy to check that

$$
\left[(L+(p-1) v)(L-v)^{p-1}-(p z)^{p}\right] V_{p, \nu}(z)=0,
$$

and the result follows by differentiating (19) with respect to $\nu$, setting $\nu=0$, and observing that $\left.U_{p}(z)=-\partial V_{p, \nu}(z) /\left.\partial v\right|_{v=0}.\right\}$ Now

$$
V_{p}(z) \sim p^{-1 / 2}(2 \pi z)^{(1-p) / 2} \exp (p z)
$$

is the dominant solution of (18) as $z \rightarrow+\infty$. By analyzing the asymptotic behavior of the subdominant solutions we obtain

$$
\left|U_{p}(z) / V_{p}(z)\right|=O(\exp (-c(p) z)) \quad \text { as } z \rightarrow+\infty,
$$

where $c(p)=p(1-\cos (2 \pi / p))$. Thus, the error in (17) for integer $p \geqslant 2$ is $O(\exp (-c(p) n))$ as $n \rightarrow \infty$. Since $c(2)=4, c(3)=4.5$, and $c(p) \leqslant 4$ for $p \geqslant 4$, only the case $p=3$ is worth considering as a computational alternative to method B1 (i.e. the case $p=2$ ). 
5. Computational Results. $\gamma$ was computed to more than 30,100 decimal places using method B1 and a multiple-precision arithmetic package [9] on a Univac 1100/42. Three independent computations were performed, with $n=17,332$ (using base 10,000 and 7,527 digits), $n=17,357$ (base 65,535 and 6,260 digits), and $n=17,387$ (base 65,536 and 6,271 digits). All three agreed to 30,100 decimal places, and the last two agreed to 30,141 decimal places. The computer time required for each computation was about 20 hours, much the same as for the 20,700 decimal place computation [7] using Sweeney's method on the same machine.

We also computed $G=\exp (\gamma)$ to more than 30,100 decimal places using the exponential routine in Brent's package [9] (with base 65,536 and 6,260 digits), and verified it by computing $\ln (G)$ by the Gauss-Salamin algorithm [6] (with base 10,000 and 7,550 digits). The rounded 30,100D values of $\gamma$ and $G$ are given in [10].

The first 29,200 partial quotients in the regular continued fractions for $\gamma$ and $G$ were computed and verified as in [7], [8]. Statistics on the distributions of the first 29,000 partial quotients are given in Table 1, with notation as in [7, Table 2]. A chisquared test did not show any significant difference (at the $5 \%$ level) between the actual distributions and the distribution predicted by the Gauss-Kusmin theorem [13]. A table of the first 29,000 partial quotients for $\gamma$ and $G$ is given in [11].

TABLE 1

Distribution of first 29,000 partial quotients for $\gamma$ and $G$

\begin{tabular}{crrr}
\hline$n$ & $\begin{array}{c}\text { number of } \\
q_{i}(\gamma)=n\end{array}$ & $\begin{array}{r}\text { number of } \\
q_{i}(G)=n\end{array}$ & $\begin{array}{c}\text { expected } \\
\text { number }\end{array}$ \\
\hline 1 & 12112 & 11992 & 12036.1 \\
2 & 4809 & 4875 & 4927.8 \\
3 & 2791 & 2760 & 2700.2 \\
4 & 1727 & 1757 & 1707.9 \\
5 & 1181 & 1168 & 1178.6 \\
6 & 867 & 848 & 862.7 \\
7 & 642 & 716 & 658.9 \\
8 & 497 & 520 & 519.7 \\
9 & 420 & 417 & 420.5 \\
10 & 346 & 335 & 347.2 \\
$11-20$ & 1624 & 1729 & 1694.1 \\
$21-50$ & 1148 & 1103 & 1133.9 \\
$51-100$ & 411 & 390 & 400.2 \\
$101-1000$ & 378 & 349 & 370.4 \\
$>1000$ & 47 & 41 & 41.8 \\
\hline
\end{tabular}

From the continued fractions for $\gamma$ and $G$ we can improve the Theorem of [7, Section 7], where the lower bound on $|Q|$ was $10^{10,000}$.

THEOREM 1. If $\gamma$ or $G=P / Q$ for integers $P$ and $Q$, then $|Q|>10^{15,000}$. 
Acknowledgement. This work was initiated while the first author was visiting the Department of Electrical Engineering and Computer Sciences at the University of California, Berkeley. The computations were performed at the Australian National University.

Department of Computer Science Australian National University

P. O. Box 4

Canberra, A. C. T. 2600, Australia

Lawrence Berkeley Laboratory

University of California

Berkeley, California 94720

1. M. ABRAMOWITZ \& I. A. STEGUN, Handbook of Mathematical Functions with Formulas, Graphs, and Mathematical Tables, National Bureau of Standards, Washington, D. C., 1964.

2. M. BEELER, R. W. GOSPER \& R. SCHROEPPEL, "Hakmem," Memo No. 239, M.I.T. Artificial Intelligence Lab., Cambridge, Mass., 1972, pp. 70-71.

3. W. A. BEYER \& M. S. WATERMAN, "Error analysis of a computation of Euler's constant," Math. Comp., v. 28, 1974, pp. 599-604. MR 49 \#6555.

4. W. A. BEYER \& M. S. WATERMAN, "Decimals and partial quotients of Euler's constant and In 2," UMT 19, Math. Comp., v. 28, 1974, p. 667. Errata: Math. Comp., MTE 549, v. 32, 1978 , pp. 317-318.

5. R. P. BRENT, "The complexity of multiple-precision arithmetic," Complexity of Computational Problem Solving (R. S. Anderssen and R. P. Brent, Eds.), Univ. of Queensland Press, Brisbane, 1976, pp. 126-165.

6. R. P. BRENT, "Multiple-precision zero-finding methods and the complexity of elementary function evaluation," Analytic Computational Complexity (J. F. Traub, Ed.), Academic Press, New York, 1976, pp. 151-176. MR 52 \#15938, 54 \#11843.

7. R. P. BRENT, "Computation of the regular continued fraction for Euler's constant," Math. Comp., v. 31, 1977, pp. 771-777. MR 55\#9490.

8. R. P. BRENT, " $\gamma$ and $\exp (\gamma)$ to $20700 \mathrm{D}$ and their regular continued fractions to 20000 partial quotients," UMT 1, Math. Comp., v. 32, 1978, p. 311.

9. R. P. BRENT, "A Fortran multiple-precision arithmetic package," ACM Trans. Math. Software, v. 4, 1978, pp. 57-70.

10. R. P. BRENT, "Euler's constant and its exponential to 30,100 decimals," Computing Research Group, Australian National University, Sept. 1978. Submitted to Math. Comp. UMT file.

11. R. P. BRENT \& E. M. MCMILLAN, "The first 29,000 partial quotients in the regular continued fractions for Euler's constant and its exponential," submitted to Math. Comp. UMT file.

12. J. W. L. GLAISHER, "History of Euler's constant," Messenger of Math., v. 1, 1872, pp. 25-30.

13. A. YA. KHINTCHINE (A. JA. HINČIN), Continued Fractions, 3rd ed., (English transl. by P. Wynn), Noordhoff, Groningen, 1963. MR 28 \#5038.

14. G. F. B. RIEMANN, “Zur Theorie der Nobili'schen Farbenringe," Poggendorff's Annalen der Physik und Chemie, Bd. 95, 1855, pp. 130-139. (Reprinted in Bernhard Riemann's Gesammelte Mathematische Werke und Wissenschaftlicher Nachlass, Teubner, Leipzig, 1876, pp. 54-61.)

15. A. SCHÖNHAGE \& V. STRASSEN, "Schnelle Multiplikation grosser Zahlen," Computing, v. 7, 1971, pp. 281-292.

16. D. W. SWEENEY, “On the computation of Euler's constant," Math. Comp., v. 17, 1963, pp. 170-178. MR $28 \# 3522$.

17. A. VAN DER POORTEN, "A proof that Euler missed-Apéry's proof of the irrationality of $\zeta(3), "$ Mathematical Intelligence, v. 1, 1979, pp. 196-203.

18. H. WALL, Analytic Theory of Continued Fractions, Van Nostrand, New York, 1948.

19. G. N. WATSON, A Treatise on the Theory of Bessel Functions, 2nd ed., Cambridge

Univ. Press, London, 1944. 